\title{
Playable One-Switch Video Games for Children with Severe Motor Disabilities Based on GNomon
}

\author{
Sebastián Aced López \\ Politecnico di Torino \\ Corso Duca degli Abruzzi, 24 \\ Torino, 10129 Italy \\ Tel.: +39011090 7191 \\ Fax: +390110907099 \\ E-mail: sebastian.acedlopez@polito.it
}

\author{
Fulvio Corno \\ Politecnico di Torino \\ Corso Duca degli Abruzzi, 24 \\ Torino, 10129 Italy \\ Tel.: +390110907053 \\ Fax: +390110907099 \\ E-mail: fulvio.corno@polito.it
}

\author{
Luigi De Russis \\ Politecnico di Torino \\ Corso Duca degli Abruzzi, 24 \\ Torino, 10129 Italy \\ Tel.: +39011090 7170 \\ Fax: +390110907099 \\ E-mail: luigi.derussis@polito.it
}

\begin{abstract}
Being able to play games in early years is very important for the development of children. Even though, children with physical disabilities encounter several obstacles that exclude them from engaging in many popular games. In particular, children with severe motor disabilities that rely on one-switch interfaces for accessing electronic devices find dynamic video games completely unplayable. In this paper we present the development and evaluation of GNomon: a framework, based on the NOMON interaction modality, that enables the creation of dynamic one-switch games for children with severe motor disabilities. The framework was designed following a series of guidelines elicited in close collaboration with a team of speech therapists, physiotherapists and psychologists from one of the Local Health Agencies in Turin, Italy. Likewise, three mini games were developed for testing the playability of GNomon-based games. Finally, we conducted a series of trials with 8 children with severe motor disabilities assisted by the health agency, in which we found that all of them enjoyed playing the GNomonbased mini games and that 7 of them were able to interact and play autonomously.
\end{abstract}

Keywords-accessible games, one-switch interaction, assistive technology, children with disabilities, single switch selection.

\section{INTRODUCTION}

Play is the leading source of children's development [1], thus the ability to play games in their various forms is very important during childhood. Nowadays, one of the most popular forms of games among children are video games (see the United States and Europe statistics reported in [2] and in [3], respectively). Video games differ from other type of games because of their interactiveness; video games offer dynamic game mechanics, a wide variety of game genres and sophisticated interfaces that make them a unique entertaining experience to actively engage in. Moreover, these games not only entertain, but benefit players in cognitive, motivational, emotional and social aspects in ways never before afforded, according to [2].

However, this is not always the case for many children with disabilities ${ }^{1}$ because they do not have the sensorial, cognitive or motor skills for accessing off-the-shelf video games. In

\footnotetext{
${ }^{1}$ Although disability is a complex multidimensional experience that poses several challenges for measurement, UNICEF [4] estimates that the $5.1 \%$ and $0.7 \%$ of children (aged 0 to 14 ) worldwide live with "moderate to severe disabilities" and "severe disabilities", respectively.
}

particular, children with motor disabilities often lack the ability to use standard input devices such as keyboard, mouse or game controllers, and thus they are usually excluded from playing video games similar to those played by their peers. Moreover, children with severe motor disabilities that rely on the use of a single switch for accessing electronic devices, find dynamic video games completely unplayable.

The problem is that dynamic video games are fast-paced games that require rapid decision-making and timely responses from the player for successful interaction; conversely, switch interaction (a.k.a. one-switch interaction) is typically based on scanning, a selection mechanism that is not intended for timedependent tasks or rapid decision-making processes. In fact, scanning requires the selectable elements to be stationary in fixed layouts and takes time to be operated, as will be explained in detail in Section II. Dynamic games, on the other hand, are characterized by the presence of elements that can move around the screen and by offering complex visual scenes which can change quickly. As a result, one-switch users have access to static, time independent and barely interactive games.

There is therefore a clear need for dynamic video games playable by children with severe motor disabilities that rely on the use of a single switch (from now on referred as the target children) as they want to play games similar to those played by their peers without disabilities, as found by Hernandez et al. in a year-long participatory study reported in [5]. Additionally, it has been shown (e.g., in [6] or [7]) how complex training environments such as video games, specifically dynamic or action ones, produce learning that transfers well beyond the training task.

The objective of this paper is twofold. First, we present GNomon, a framework that enables the creation of accessible dynamic one-switch video games. The framework was designed following a series of guidelines elicited in close collaboration with a team of speech therapists, physiotherapists and psychologists from one of the Local Health Agencies in Turin, Italy. The second goal is to report the results of the evaluation of three GNomon-based mini games with 8 target users assisted by the health agency. These results, and the associated discussion, allow us to tackle the research question of whether it is possible to develop dynamic video games playable by children with severe motor disabilities using just 
a single switch as input device.

This paper is organized as follows: Section II provides useful background concerning accessible interfaces for persons with severe motor disabilities, with special focus on those used by children, and the state of the art of video games based on one-switch interaction. Section III describes the GNomon framework, its design process and its final features, while Section IV presents the development of three mini games based on it. Section V reports the playability evaluation of the mini games with a group of 8 children with severe motor disabilities and Section VI discusses the results. Finally, the conclusions extracted from this work are presented in Section VII.

\section{BACKGROUND}

In this Section we present some relevant one-switch interfaces and alternative interaction mechanisms accessible by persons with severe motor disabilities of all ages, including children. We also review and classify several free online oneswitch games suitable for our target children.

Persons that live with severe motor disabilities caused by traumatic injuries (e.g., spinal cord injury), diseases (e.g., Lou Gehrig's disease) or congenital conditions (e.g., cerebral palsy) usually interact with computers using assistive direct selection or using scanning interfaces. Direct selection refers to the action of somehow pointing (e.g., with the finger or with the gaze) at any desired item for selecting it, whereas scanning allows the selection of one element at a time from an arrangement of selectable elements, in sequential order. Specifically, scanning works by sequentially highlighting the selectable elements (or groups of elements) and waiting a short time (i.e., scanning delay) on each of them for an input to occur. Such an input is usually the activation of a single switch, which consists in just a "click" with timing information (i.e., the moment of the click, but its duration). Finally, an element is selected if it is the one currently highlighted when the input event occurs. Although scanning is considered slower and more cognitively taxing than direct selection, generally consists of a much simpler and inexpensive set up and it requires considerably less motor control to be used efficiently (see [8] for more details about scanning as an interface method). Thus, scanning interfaces operated through single switches are very popular among our target children.

Over the years, much research has concentrated on improving one-switch selection mechanisms for enhancing the interaction capabilities of persons with severe motor disabilities of all ages. In the book of Beukelman et al. [9], the authors discuss diverse alternative access options to address complex communication needs, such as different scanning control techniques while Jennifer Angelo, in [8], systematically compares three basic modes of scanning (e.g., automatic, inverse and step) for supporting one-swtich interaction of persons with cerebral palsy. Other studies, such as [10] and [11], have focused on designing entire systems to provide one-switch users accessibility to computer applications, which is essential for participating in modern information society. In the last decade, alternative input methods for empowering one-switch users to interact with electronic devices have been explored, specially for text entry. However, the interfaces specifically designed for text entry maybe slow, and are usable by children only if they are literate. Mackay et al. proposed Dasher [12], an information-efficient text-entry interface, driven by natural continuous pointing gestures which can be carried out through single switches. Broderick and MacKay described NOMON in [13], a fast and flexible interface which serves as a substitute for any point-and-click activity on a computer screen. Belatar and Poirier presented HandiGlyph [14], an interface for mobile devices to allow users with severe motor impairments to entry text.

Alternative interfaces for one-switch users operated without the use of single switches are attracting widespread interest. Researchers such as Harada et al. conducted a longitudinal study on voice cursor control reported in [15], and a group of the University of Malaga presented in [16] a brain-computer interface paradigm that allows to select several output commands using only two mental tasks.

Unfortunately, previous works were mainly developed having in mind text entry or menu selection, contexts in which 1) selection sets are large and the occurrence of having to repeat an input is low, 2) selection sets are known and static, 3) single elements can be arranged in fixed positions to enhance selection speed and effectiveness, 4) each element has a computable probability of being the next to be selected, given the previous selections. Consequently, when the aforementioned one-switch selection mechanisms and principles are utilized to enable our target children to interact with video games, the resulting experience is often trivial, static or boring, as illustrated in the following review of several free games (games listed in a popular non-profit website ${ }^{2}$ ). The criteria for including the games in the review was that they can be played with just a two-state single switch. We identified three major categories of one-switch video games and the main barriers that prevent children to enjoy them:

1) Action/Reaction games. The goal on these simple games is to press the switch to obtain some effect (e.g., when the switch is pressed, a football player kicks a ball ${ }^{3}$ ). These games are used to teach the children how to use the switch and to associate it to a trigger for different actions. The downside of many action/reaction games is the lack of interactiveness for cognitively able children due to the fact that they cannot make any decision to affect the outcome of the game, as the effect of pressing the switch at any time is always the same.

2) Scanning based games. Most of the available one switch games (e.g., chess, memory, battleship, etc.) fall under this category. These are usually more complex than action/reaction games as scanning allows to select more than one element (or action) from an arrangement of options. However, it is not possible to interact with video games that do not have a static arrangement of selectable elements, a fixed scanning order and suitable game mechanics to allow enough time to make any selection even after a complete scan.

3) Click timing games. This category of one switch games includes those in which the player has to press the switch with high precision to perform an action in a

\footnotetext{
${ }^{2}$ http://www.oneswitch.org.uk, last visited on April 30, 2015

${ }^{3}$ http://www.specialbites.com/switch-activated-games/ penalty-shootout-cause-and-effect-switch-game.html, last visited on April 30,2015
} 
very specific moment. The problem with many of these games, such as Poto \& Cabenga ${ }^{4}$ and Strange Attractors ${ }^{5}$, is that they are not fully accessible to children with severe motor disabilities because such games require a speed and precision that these children often do not have.

To our knowledge, we are the first to address the question of whether it is possible to develop dynamic video games playable by children with severe motor disabilities using only a single switch as input device.

\section{GNOMON}

GNomon is a framework that enables the creation of accessible and dynamic one-switch video games (the first idea about it was presented as a work in progress by Aced López et al. in [17]) . It is based on the NOMON one-switch mode of interaction, which allows to select one of many elements from the screen without extra special hardware (e.g., expensive eye trackers) and without requiring them to be arranged in any particular configuration or to be stationary.

In a very inspiring publication [13], Broderick and MacKay present in detail the operation and evaluation of NOMON, therefore we report just the main aspects of its operation here:

- NOMON associates a small visible clock face to each selectable element on the screen and places it next to the element.

- Every clock has one black hand which rotates at the same speed as the other clocks' hands, but with a different phase. The clocks also have a red hand fixed at "noon".

- To select an element the user has to find the associated clock and try to press the switch, as precisely as possible, when the black hand is crossing the red hand at noon. Then, for each clock, NOMON calculates the probability of it being the intended selection, given the clicks thus far. If the probability of a clock is sufficiently high but not enough to declare it as the selection, because there are also other likely candidates, the clock faces of all candidates turn yellow and a new round starts. Finally, when the probability of one clock reaches a predefined threshold, it turns green and the associated element gets selected.

In particular, GNomon provides functionalities for creating sets of selectable game objects with associated NOMON clocks for enabling dynamic point-and-click game mechanics using a single switch. These sets can be resized at any time by adding or removing elements, which is necessary for supporting common dynamic game actions such as the creation of new items or the destruction of characters. Moreover, the framework is also very useful to make static games (e.g., scanning-based games) more challenging by allowing selectable elements to be displayed without layout restrictions.

A participatory approach was adopted for eliciting the features and accessibility guidelines of GNomon, thus we worked in close collaboration with a team of speech therapists, physiotherapists and psychologists from one of the Local Health Agencies in Turin, Italy. They actively supported us

\footnotetext{
${ }^{4} \mathrm{http}: / /$ www.potoandcabenga.com/, last visited on April 30, 2015

${ }^{5} \mathrm{http}: / /$ ominousdev.com/strange-attractors/, last visited on April 30, 2015
}

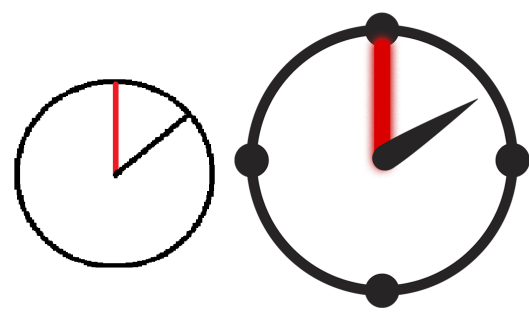

Fig. 1. This figure shows an example of the clocks used in GNomon for selecting game objects (right) and the original NOMON clock (left). The appearance of the new GNomon clock is based on the original design but with important modifications suggested by the experts: enlarging the clock, thickening all clock lines, placing marks at clock quarters, rendering more eye-catching the red noon hand and making the moving clock hand pointier.

by guiding important design choices and proposing features on behalf of the children assisted by them. In total, we conducted five meetings with the experts for testing and collecting suggestions to improve the framework. Thus, several features of GNomon, mainly related to specific accessibility issues, were adapted to follow their valuable recommendations. The recommendations are the following:

1) Providing additional indicators for facilitating interaction. In particular, four circular marks were placed in the clock quarters (the three o'clock, six o'clock and nine o'clock) to facilitate the interaction of children with long muscular latent periods (i.e., the time elapsed between the movement command and the muscle movement) by helping them with indications of when to start "preparing" themselves for pressing the switch.

2) Making the clocks more eye-catching. Game objects are usually attractive, colorful and animated, hence they tend to concentrate the attention of children. Moreover, as the clocks are just the means for selecting game objects, it is normal that the former are less striking than the latter. However, the clocks' appearance was redesigned to be as eye-catching as possible (while keeping its simplicity) to prevent the less attentive children from ignoring the clocks. In particular, the clocks were enlarged, the colors were made brighter and more contrasting, the lines were thickened and the moving clock hand was made pointier. Figure 1 shows a comparison between the classical and new appearances of the clocks.

3) Reducing the average speed of rotation of the moving clock hands and making it customizable. The rotation period of the clock hands ranges from 1 to 10 seconds, to allow children with long muscular latent periods to enjoy GNomon based games. The rotation speed can be set and changed easily by the children caregivers.

4) Giving auditory and visual feedback when a clock is selected. Besides the specific feedback and the actions triggered in each game when a clock is selected, the clock itself changes color and a sound is played.

The final implementation of the GNomon framework incorporates our custom extended $\mathrm{C \#}$ versions of the original NOMON Python libraries into a Unity $2 \mathrm{D}^{6}$ plugin.

\footnotetext{
${ }^{6}$ http://unity3d.com/unity/2d-3d, last visited on April 30, 2015
} 


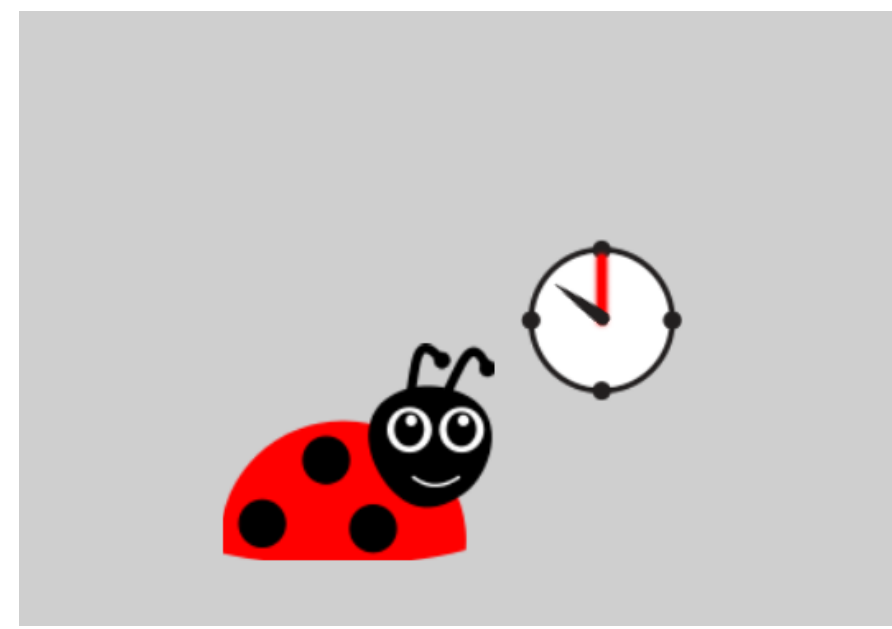

Fig. 2. The figure shows a red ladybug and its associated clock during a One Switch Demo game.

\section{THE GAMES}

Three prototype games ${ }^{7}$ with different degrees of difficulty which are fully playable with just one switch were designed in collaboration with the Regional Health Agency. Here we present them in order of difficulty: One Switch Demo, One Switch Ladybugs and last One Switch Invaders.

\section{A. One Switch Demo}

The first game is One Switch Demo, a very simple action/reaction video game that allows children to make a red ladybug jump when they select the clock associated to it. When the ladybug is selected correctly and it jumps, it also produces an auditory feedback to increase the children's reward. There are no scores or time constraints of any kind because the game has been designed mainly to explain how clock selection works. The aesthetics, as the objective of the game, are very simple to help the children with visual and attention difficulties to stay focused on the game mechanics. Figure 2 shows a screenshot of the game.

\section{B. One Switch Ladybugs}

The second game is One Switch Ladybugs, another simple mini game that allow children to make one of four ladybugs jump. Each ladybug has a different color and provides a unique auditory and visual feedback when it is successfully selected. There are no scores or time constraints of any kind, but unlike the first game the aesthetics are richer and there is background music playing in loop. The objective of the game is the same as in the previous game: to make a ladybug jump, with the difference that in this case, the children have to first choose the ladybug they want to make jump. Figure 3 shows a screenshot of the game.

\section{One Switch Invaders}

The third game is definitely more complex than the first two and is called One Switch Invaders. It is an engaging dynamic

\footnotetext{
${ }^{7}$ Downloadable at http://elite.polito.it/gnomon-games, last visited on April 30,2015
}

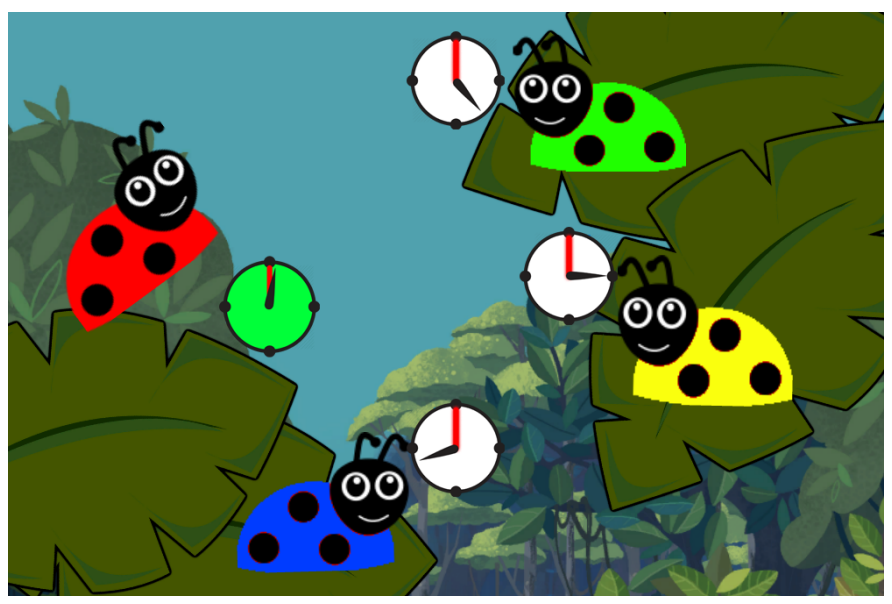

Fig. 3. The figure shows the red ladybug (top left) jumping while it is being selected during a One Switch Ladybugs game.

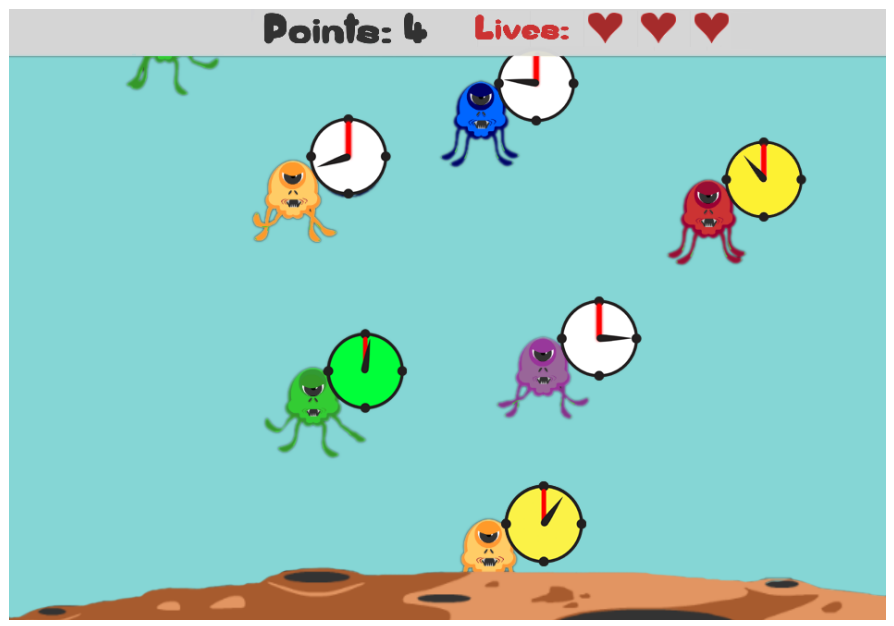

Fig. 4. The figure shows six aliens falling down the screen while the green one (at the left) is being selected during a game of One Switch Invaders.

one-switch game that does not require accurate timing or clicking precision to be played. This is a game without a fixed layout, in which multiple selectable elements (aliens), moving down across the screen, have to be selected with a time constraint (i.e., before they touch the ground). The implementation of One Switch Invaders is not feasible by applying scanning interfaces because the elements are not static in predefined layouts and it is not possible to establish a scanning order without negatively affecting the game mechanics.

The game objective is to score points by eliminating the aliens before three of them touch ground. There are aliens of five different colors which constantly fall down the screen at a bounded random speed. Each alien is associated to a clock to enable its selection and is generated in a random position at the top of the screen. Figure 4 shows a screenshot of the game.

\section{Design Considerations}

The design of the mini games (specially One Switch Ladybugs and One Switch Invaders) take into account some of the valuable design recommendations from Hernandez et al. in 
[5] for playable action-oriented video games for children with cerebral palsy:

1) Simplify level flow, reducing the number of decisions players need to make and reducing the demands on visualspatial reasoning. In One Switch Ladybugs, this is taken into account by presenting only four ladybugs which can perform just one action (jump). Although in One Switch Invaders the player has many aliens to eliminate, the decision of which one to eliminate first is simple: the lowest one, because the goal is to keep them out of the ground and all them fall with an equal and constant speed.

2) Reduce consequences of errors, ensuring that errors due to difficulties completing rapid or time-sensitive actions do not impair fun. While in One Switch ladybugs there is no error penalty at all, in One Switch Invaders players have three lives before they lose. Moreover, if a player misses the selection of an alien, she has more opportunities to kill it as the falling time of its associated clock is enough for allowing several clock hand revolutions before the alien touches the ground.

3) Limit available actions, reducing the number of decisions players need to make, and enabling a simpler control scheme. The control scheme of GNomon-based games consists of just one action: to press the switch. The only decisions that the players have to make, besides from when to press the switch for selecting the desired clock are: which ladybug jumps next in One Switch Ladybugs and which alien is better to kill next in One Switch Invaders.

4) Remove the need for precise positioning and aiming, reducing the demands on manual ability and visual-motor integration. This is part of the selection process itself. The player does not have to press the switch precisely when the clock hands are together for the interface to work. Moreover, if there is ambiguity between two or more elements, their associated clocks turn yellow to indicate it.

5) Make the game state visible, reducing the need for attention to gameplay, and reducing the need for visual spatial reasoning to deduce game state. Only One Switch Invaders has a state, displayed at the top bar, which consists in the number of remaining lives and points reached.

Finally, we also present the main recommendations made by the Regional Health Agency experts regarding the prototype games:

- The appearance of each game object (ladybugs or aliens) must be unique. This helps the children to remember the game object that they are trying to select if they lose track of it. For the ladybugs, this was achieved by assigning them four different colors and placing them in the four quadrants of the screen. However, in the case of the aliens this is not possible because there is not a predefined number of aliens that can be on the screen at the same time. Therefore, the solution adopted in the second game was choosing five colors to be sequentially assigned to each new alien. In this way, children that lose track of their game object can retrieve it without being confused with another near object that looks the same.
- Different game objects have to produce different visual and/or acoustical feedbacks when selected.

- Although aliens can fall with different speeds, the maximum falling speed has to be bounded by a maximum value set by the player or the caregiver. However, for evaluation pourposes, in this version all aliens fall at the same constant speed.

- In the One Switch Invaders game, the aliens wait until the first "click" to start falling. In this way, the player can observe the clocks and familiarize with the speed of rotation of the clock hands before trying to select one of them.

\section{Evaluation}

Following the design and implementation phase of GNomon and the mini games, an evaluation of these was conducted in collaboration with the group of experts of the Regional Health Agency. The research question to answer was whether it is possible to develop dynamic video games that are playable by children with severe motor disabilities using just single switch as input device. In particular, we were interested in determining if GNomon can be used for such a purpose. For this evaluation, we consider a game playable (suitable for being played) if it is usable and fun for the player (based on the concept and characteristics of playability presented in [18]). Hence, we assess the following properties of playability of the GNomon-based mini games through a series of eight tests:

- Learnability: How easy is for the children to understand and successfully start to use a GNomon-based interface after it has been explained?

- Effectiveness: Does a GNomon-based interface actually support the children in achieving the various goals of the game?

- Errors: How many times the element selected does not match with the element that the children had in mind to select?

- Satisfaction: Do children like to play video games with a GNomon-based interface?

The tests were conducted in Italian at the Regional Health Agency with children between 4 and 14 years old with severe motor disabilities, who already consistently use one-switch interfaces. The sessions were carried out at the facilities of the Health Agency, a known environment for the children, to avoid making some children anxious about being in an unfamiliar place. An expert speech therapist (tester) held the sessions and two observers carefully took notes during these. It was decided to have an expert speech therapist as the tester simply because she knows how to communicate more effectively with the children (in particular with non-verbal children) than HCI researchers. Similarly, the reason for having just two observers during the tests, (one expert from the Health Agency and one HCI researcher) was to prevent some children from distracting and being uncomfortable in the presence of many people.

\section{A. Set-up and Materials}

All the tests were video recorded, with the authorization of the children's parents and hiding the children faces, in order to allow researchers to observe simultaneously the mini games 
TABLE I. MATERIALES USED FOR CARRYING OUT THE TESTS

\begin{tabular}{l} 
Materials \\
\hline Intel i7 laptop with 8GB of RAM running 64-bit Windows 7 \\
One Switch Demo game \\
One Switch Ladybugs game \\
One Switch Invaders game \\
Helpibox 16 interface \\
Big Red 5-inch switch \\
Logitech Orbit webcam \\
Adjustable school table \\
Alternative and Augmentative Communication (AAC) tables \\
21” VGA monitor
\end{tabular}

being played in a 21 " screen and the children while they were pressing the switch, for later analysis. The switch utilized for playing was a Big Red 5-inch mechanical switch activated with the hand or with the head, depending on the capabilities of each child. The switch was connected to a Intel i7 laptop with 8GB of RAM running Windows 7 . The interface between the switch and the computer was a Helpibox 16. In the case of non verbal children, it was also necessary to use Alternative and Augmentative Communication (AAC) tables to allow them to answer the tester questions, in particular these tables contained the colors of ladybugs, the yes/no answers and the faces to indicate "like" or "dislike". Table I summarizes the materials used for carrying out the tests.

\section{B. Participants}

The participants of this study are 8 children, 7 boys and 1 girl, with severe motor disabilities. All of them are between 4 and 14 years old and rely on the use of single switch interfaces to access electronic devices. The severe motor impairments of the children have different causes, which in some cases entail cognitive disabilities as well (as in the case of some children with Cerebral Palsy). See Table II for a detailed summary of the participants.

Given the fact that the participants are children which have special communication needs due to their disabilities, it was not possible for the tester to conduct the test in the exact same way for every child. Inevitably, for some children the therapist had to explain the requested tasks in more detail and avoided to ask open questions at the final questionnaire. Nevertheless, the results are acceptable as the contents of the information provided (and requested) by the therapist to (and from) the participants was essentially the same. Only its presentation was adapted to suit better each child capabilities.

\section{Procedures}

As mentioned before, each session was conducted and video recorded at the Regional Health Agency facilities, in Turin, by an expert speech therapist with two observers: another therapist and a HCI researcher. The recruitment of the participants was straightforward since the Health Agency proposed and gathered the children and helped us to fix the appointments for the tests. The criteria for selecting the participants was that they had to be children between 4 and 14 years old, which use a single switch for accessing electronic devices or that will do it in the near future due to degenerative conditions.
The sessions were held in Italian and lasted between 7 and 18 minutes, depending on various factors such as the cognitive or visual difficulties of the children taking the test, their fatigue and how much they wanted to play each game. The sessions, although in a flexible way, were structured in three parts. In the first part, the tester explained the scope of the first game (i.e., to make the single ladybug jump) and how to accomplish it. The tester demonstrated two times the selection mechanism by making the ladybug jump. Then, the child tried to make the ladybug jump and could play for 2 minutes maximum. In the second part, the speech therapist presented the One Switch Ladybugs game to the child by saying that it was played in the same way as before, but explaining that this time it was necessary to identify which of the four ladybugs make to jump and to concentrate on the clock next to it. As before, the tester exemplified twice. Then, he asked the children to make a different ladybug jump following a standard sequence 1) green, 2) red, 3) yellow, 4) blue. Finally, the child was asked to choose a ladybug to make it jump and to say it out loud (the non-verbal participants used an AAC table for this task) before selecting it. At the end of this part of the test, the child could play freely for 3 minutes maximum. The last part of the evaluation consisted in playing the One Switch Invaders game but it was carried out only with participants P5, P6 and P8. This part of the test was not proposed to the other participants because during the preparation of the evaluation phase, the experts considered that it would be above the cognitive or visual-attentive capabilities of children P1, P2, P3, P4 and P7, thus no reliable results could be expected from them. However, when the third part of test was conducted, it followed a similar scheme than the one adopted for the other parts of the test: the objective of the game was explained to the children, then the tester demonstrated twice how to kill the aliens and finally the children could play freely for 5 minutes maximum.

At the end of each game, three questions were asked to the participants:

1) Did you like the game?

2) What did you like the most?

3) What did you not like?

Likewise, at the end of the entire session, the children were also asked the following questions:

1) Did you remember how to select the elements while you were playing?

2) Was it difficult to select the elements?

3) Did you have fun?

At the end of all the tests, the recordings were carefully transcribed for further analysis, as well as the observation notes taken by the observers. Moreover, we also logged data from each test; in particular, we collected information about: the rotation speed of the clocks, the falling speed of the aliens (in the One Switch Invaders game), the number of clicks performed and the time of each one of them, the selection of a ladybug in the One Switch Ladybugs game and the final score in the One Switch Invaders game.

\section{Observations}

Before discussing the obtained data, some general observations are presented, in order to provide contextual information useful for understanding the following sections of this paper. 
TABLE II. PARTICIPANTS WITH DETAILS ABOUT AGE, IMPAIRMENTS, DIAGNOSIS AND BODY PART USED FOR OPERATING THE SWITCH

\begin{tabular}{|c|c|c|c|c|c|}
\hline Participant & Age (years) & Gender & Impairments & Diagnosis & Switch activation \\
\hline P1 & 4 & Male & Non-verbal with spastic quadriplegia with dystonia & Cerebral Palsy & Head \\
\hline $\mathrm{P} 2$ & 4 & Female & Postural hypotonia & Aicardi-Goutières syndrome (AGS) & Head \\
\hline P3 & 4 & Male & Spastic quadriplegia, cognitive and communication difficulties & Cerebral Palsy & Hand \\
\hline P4 & 6 & Male & Spastic quadriplegia, strabismus and cognitive difficulties & Cerebral Palsy & Hand \\
\hline P5 & 7 & Male & Muscular dystrophy & Spinal Muscular Atrophy (SMA) & Hand \\
\hline P6 & 7 & Male & Moderate cognitive difficulties & Down syndrome with right-side hemiparesis & Hand \\
\hline P7 & 8 & Male & Cognitive, visual and coordination difficulties. Spastic quadriplegia. & Cerebral Palsy & Hand \\
\hline P8 & 14 & Male & Muscular dystrophy & Spinal Muscular Atrophy (SMA) & Hand \\
\hline
\end{tabular}

There were minor difficulties while testing the games with the children at the Regional Health Agency mainly related to the children's mood. In general, the youngest children with cognitive or attention difficulties (P1 and P3) became tired by the end of the first game (i.e., the one with a single ladybug) and faced the rest of the test with unfavorable attitude. In particular, P1 (which is a non-verbal child) started to stare at the door when the tester asked him for the fourth time to select a ladybug during the second game, indicating that he wanted to leave the room. Similarly, P3 at the end of the second game, did not want to freely play as he wanted at the end of the first game. Nevertheless, the experts that regularly assist these children agreed that it was normal that they were tired after trying new experiences that require them attention and concentration.

Another slightly problematic situation happened with P2, a 4-years-old girl, as she was not being cooperative because she wanted her mother in the room. The tester and the observers decided to call in the mother in order to calm the child. However, although the child calmed down, she was very distracted by continuously looking at her mother and she was not able to perform at her best in the test.

\section{RESULTS AND DISCUSSION}

In this section we present the data and information collected during the playability tests, along with their analysis and the derived research findings. First, we present the observations made during the tests along with Table III, which summarizes them. Secondly, we asses the learnability, effectiveness, errors and satisfaction of the mini games stemming from the aforementioned table and from the careful analysis of the video recordings of each test. Lastly we attempt to answer the research question of whether it is possible to develop dynamic video games playable by children with severe motor disabilities using just a single switch as input device.

The tests lasted in average 13 minutes and all participants, except P7, played at least the first two games (One Switch Demo and One Switch Ladybugs). What happened with P7 is that he could not make the single ladybug jump in the One Switch Demo. He was not even trying to make the ladybug jump as he was distracted by watching the clock hand rotating or looking at the table, hence the tester decided to stop the test. On the other hand, P5, P6 and P8 were able to play satisfactorily One Switch Invaders.

The errors were higher in the case of children with cognitive or attention impairments than in the case of children with just motor disabilities, as expected. In particular, P3 and
P4 were facilitated by hiding two of the four ladybugs from them in an initial phase. In such a way, the tester limited the visible options at the beginning of the free selection phase during the One Switch Ladybugs game, to help the children to concentrate in the ladybug they wanted to select. By contrast, P5 and P8 had no errors when they tested all the three mini games, moreover they asked to play One Switch Invaders again but with a faster falling speed (i.e., the falling speed is the speed at which the aliens fall down the screen).

All participants but one (P1) answered that they enjoyed and had fun playing the mini games. However, the negative answer of P1 to the question Did you have fun? is not reliable because it was asked at the end of the test when he was very tired, moreover it is in contrast with what he expressed at the end of the first game when he said he liked the game. It is also in contrast with the notes taken by one of the observers which reported that the child was "continuously smiling" while he was playing.

The rotation speed of the clock hands was initially determined by the tester on the basis of the previous knowledge that she had about each participant. However, when necessary, the rotation speed was modified after the first mini game. The rotation speed is expressed as an integer between 1 and 20, where 1 represents a rotation period of 10 seconds and 20 represents a rotation period of 1 second. Setting the right rotation speed was not an easy task, especially when the participants had attention difficulties because setting a fast speed increases the difficulty of successfully selecting the intended ladybug, while a slow speed gave some participants enough time to be distracted and forget about the selection task.

In Table III we summarize information regarding the following aspects: the number of games actually played by each participant, the error ratio in the One Switch Ladybugs game (i.e., the number of selections that were not the intended selection by the participant), clock hand rotation speed, overall duration of the test, the answer of each participant to the final question Did you have fun? and the most relevant observers' notes.

\section{A. Playability assessment}

From the data reported above we now assess the learnability, effectiveness, errors and satisfaction of the GNomon-based mini games in order to determine their playability.

1) Learnability: In order to asses the learnability of these GNomon-based games we have to determine how easy is for 
TABLE III. TEST OBSERVATIONS

\begin{tabular}{|c|c|c|c|c|c|c|}
\hline Participant & Games played & Test duration & Error ratio & Rotation speed & Was fun? & Observers' notes \\
\hline P1 & 2 & $14 \mathrm{~min}$ & $55 \%$ & 5 & NO & $\begin{array}{l}\text { The observers noted that the children understood the game as well as } \\
\text { the selection mechanism. However for activating the switch he had to } \\
\text { turn the head left, thus loosing the screen out of sight and dropping } \\
\text { the attention. He was smiling while playing at the beginning, but then } \\
\text { he was tired and wanted to leave. }\end{array}$ \\
\hline $\mathrm{P} 2$ & 2 & $15 \mathrm{~min}$ & $50 \%$ & 7 & YES & $\begin{array}{l}\text { She understood the mechanism and was able to wait for the clock hand } \\
\text { to select the ladybug she wanted, however her emotional instability and } \\
\text { her dependency on the her mother hindered a better performance. }\end{array}$ \\
\hline P3 & 2 & $18 \mathrm{~min}$ & $53 \%$ & 8 & YES & $\begin{array}{l}\text { He had difficulties concentrating on the tasks proposed by the tester, } \\
\text { so he was facilitated by limiting the ladybugs to two at the time. In } \\
\text { this way, the child learned to first decide which ladybug he wanted to } \\
\text { make jump and then to select it. When the time for freely playing the } \\
\text { second game was over, he yelled "More" twice, thus he was allowed } \\
\text { to play more after the test. }\end{array}$ \\
\hline $\mathrm{P} 4$ & 2 & $11 \mathrm{~min}$ & $62 \%$ & 6 & YES & $\begin{array}{l}\text { The child did well when there was only one ladybug (i.e., during the } \\
\text { first game), however he had the worst performance of the group in the } \\
\text { second game. He had difficulties locating and staring the ladybug he } \\
\text { was asked (or that he freely wanted) to make jump. He expressed it } \\
\text { in these words: "It was difficult whit four (ladybugs) to make jump } \\
\text { the one I wanted. I liked more the first game with just one (ladybug)" }\end{array}$ \\
\hline P5 & 3 & $10 \mathrm{~min}$ & $0 \%$ & 10 & YES & $\begin{array}{l}\text { He did not have any error during the test. He played One Switch } \\
\text { Invaders for the first time with a clock hand rotation speed of } 10 \text { and } \\
\text { a falling speed of aliens of } 8 \text {. He decided to stop playing at } 40 \text { points } \\
\text { and asked to play again but with a rotation speed of } 12 \text { and a falling } \\
\text { speed of } 10 \text {. Then he lost after making } 17 \text { points. }\end{array}$ \\
\hline P6 & 3 & $15 \mathrm{~min}$ & $29 \%$ & 8 & YES & $\begin{array}{l}\text { Even if he started activating the switch at random during the first } \\
\text { game, in the One Switch ladybugs game he did well. He presented } \\
\text { good hand-eye coordination and he was very attentive at the indications } \\
\text { of the tester. When he did not want to play more the second game he } \\
\text { tapped repeatedly the switch. He played the third game initially with } \\
\text { rotation and falling speeds of } 8 \text { and } 2 \text {, respectively. Then the tester } \\
\text { increased the falling speed to } 5 \text {, as he "wiped" the screen of aliens } \\
\text { and started to be bored. Finally, the tester increased even more the } \\
\text { falling speed up to } 10 \text {. The game was over when he tapped repeatedly } \\
\text { the switch again, indicating that it was enough for him. }\end{array}$ \\
\hline P7 & 1 & $7 \mathrm{~min}$ & - & 6 & YES & $\begin{array}{l}\text { For this participant we have no error rate relative to the One Switch } \\
\text { Ladybugs game, as he did not play it. The tester stopped the test with } \\
\text { him as he was totally distracted (e.g., he was looking at table or the } \\
\text { button) and was not trying to perform the tasks for the test. He only } \\
\text { activated the switch } 5 \text { times in } 6 \text { minutes, making the ladybug jump } \\
\text { just } 2 \text { times during the One Switch demo Game. }\end{array}$ \\
\hline P8 & 3 & $15 \mathrm{~min}$ & $0 \%$ & 10 & YES & $\begin{array}{l}\text { He had no errors at all during the test. He is the child with the most } \\
\text { severe motor disabilities which took the test, yet he has no cognitive } \\
\text { difficulties at all (just as participant P5). He enjoyed really much } \\
\text { playing the games, specially the third game and said that he had never } \\
\text { played a similar game. When the test finished, he asked if he could } \\
\text { play again One Switch Invaders, but faster (he passed from } 10 \text { to } 15 \\
\text { and a from } 8 \text { to } 10 \text {, of rotation and falling speeds respectively). He } \\
\text { played for one minute before loosing and scored } 17 \text { points. One of the } \\
\text { observers noticed him tense when many aliens were near the ground } \\
\text { and he could not avoid loosing one life. }\end{array}$ \\
\hline
\end{tabular}

the children to understand and successfully start to use the GNomon-based interface after it has been explained.

It took, in average, 2 activations of the switch for the children to understand the mechanism and to be able to consistently make the single ladybug jump. There were cases (P5 and P8) in which the participants understood and were able to use the GNomon selection mechanism immediately, but in other cases (P7) it could not even be determined if the mechanism was comprehended.

Two of the questions at the end of each test helped us to determine if the selection mechanism was difficult to grasp: Did you remember how to select the elements while you were playing? and Was it difficult to select the elements?. Not all the children were able to answer the first question because it was very difficult for their cognitive and communicative skills, but all of them (of those who played at least two games) agreed that it was not difficult to select the elements. Even
P4 said that it was easy to select the single ladybug, but he emphasized that selecting one of four ladybugs was not an easy task. Moreover, the observers agreed that the children understood how the selection mechanism works, even though some of them had trouble to operate it by themselves.

The results presented here suggest that a game with a GNomon-based interface is learnable by children with severe motor disabilities, especially if they do not have additional cognitive or visual impairments.

2) Effectiveness: Here we determine whether making games based on a GNomon-based interface is effective. For this, we assessed qualitatively (i.e., by analysing the notes taken by the observers and the video recordings) if the participants were able to play and to achieve the goals of the diverse games, utilizing the interface. Since the purpose of playing the presented mini games is to entertain, their effectiveness is highly related to the players' satisfaction. Similarly, it is also 
heavily dependant on how much it is possible for a player to make progress and interact with the games, considered his impairments and the errors derived from these. In fact, the evidence suggests that games and the interaction modality were effective, since it was certainly possible for 7 out of the 8 participants to play and have fun while achieving the games' goals, despite of the errors. Even participant P7, who could just play One Switch Demo, was able to interact with the game and the single ladybug through the GNomon-based interface while having fun. The reason for him not being able to play the second game is due to the fact that GNomon was not designed for making games for children with also visual and cognitive impairments.

3) Errors: The error ratio was measured during the second game by counting the number of actual selections that were not the intended selection by the participant. The error ratio was around $50 \%$, in average and without considering the outstanding cases of P5 and P8. Although this significant error ratio contrast with the fact that the participants had already learned the selection mechanism by this phase of the test, it is explained by the difficulties that some participants have to plan the movement and by the long latent periods (i.e., the time elapsed between the movement command and the muscle movement) they have.

However, considering that this was the first time for the participants to play these games and also that they did it for just 13 minutes (in average), it is very likely that after some training and prolonged exposure to GNomon-based interfaces the error ratio reduces considerably. In fact, the $29 \%$ error ratio of P6 (lower than the average) is given almost exclusively by the first third of the One Switch Ladybugs game since he dramatically improved during the test when he concentrated.

On the other hand, the performances of P5 and P8 are remarkable, not only because their error ratio during the second game were null, but also because they performed as well in the One Switch Invaders game. They even asked to play it again with faster settings, which indicates that dynamic games maybe very enjoyable by children with severe motor disabilities such as spinal muscular atrophy (SMA).

4) Satisfaction: Since the players' satisfaction is a very important property for a game to be playable, we assessed the satisfaction experienced by the participants as the result of playing the GNomon-based mini games. For this purpose, we asked the participants at the end of each game three questions: Did you like the game?, What did you like the most? and What did you not like?. Moreover, the tester asked them at the end of the test if they had had fun.

Although not every child was able to answer the questions What did you like the most? and What did you not like? due to cognitive or communicative impairments, 7 out of 8 participants answered affirmatively to the last question of Did you have fun?. However, the negative answer from participant (P1) is not reliable, as explained before.

The children that were able to answer the question What did you like the most? (P5, P6 and P8) said that they liked how the ladybugs jump and also the ladybug moving around. For the One Switch Invaders game, they answered that they liked the falling aliens and to be able to kill them. These answers suggest that the dynamism of the games, in particular in the case of the last game, was really appreciated by the players as something new that they were able to try.

Other children expressed their satisfaction while playing the games in diverse ways that were noticed and written down by the observers. This is the case of the participant P3, which yelled "More, more" when the tester announced him that the time for freely playing One Switch Ladybugs was over. Similarly, participant P2 indicated through an AAC table that she wanted to continue playing the second game when the tester gave her the chance to stop due to her apparent lack of interest. P5 and P8 also asked, on their own initiative, if they could play more at the One Switch Invaders game after finishing the test, which clearly indicates that they enjoyed playing the game.

Consequently, it is evident that engaging in the GNomonbased games was not only considered satisfactory but also fun by the children during the tests. Moreover, the children that had the cognitive and visual capabilities to play a dynamic game such as One Switch Invaders indicated that was the one they liked the most.

\section{B. Addressing the Research Question}

The results indicate that GNomon-based interfaces are useful to make playable one-switch video games. The playability, assessed through the properties of learnability, effectiveness, errors and satisfaction of the GNomon-based mini games tested in this study was adequate and very promising. In particular, 7 out of 8 participants learned and were able to use the GNomon-based interface to play the games. All of them had fun.

Moreover, the evidence suggests that GNomon is not only useful as an alternative to classic scanning one-switch video games, but that it might allow the development of new types of one-switch games, dynamic and time-dependent, such as One Switch Invaders, that are playable and very enjoyable by one-switch users, especially in the case of those which do not have cognitive impairments. All the participants who tried One Switch Invaders (3 out of 8 ) indicated that it was the funniest of the 3 mini games, and also that they had never played anything similar. Participant P8 asked if he could have the One Switch Invaders game for playing it again at home.

\section{CONCLUSION}

This paper proposes GNomon, a framework that enables the creation of one-switch video games for children with severe motor disabilities. It also presents the design and implementation of three mini video games with different characteristics, based on GNomon. The design of the framework and the mini games was carried out in collaboration with a team of experts from one of the Local Health Agencies in Turin, Italy. Moreover, we report the playability evaluation of the three mini games with 8 children with severe motor disabilities from which we assessed the learnability, efficiency, errors and satisfaction of GNomon based games.

The results of this work are encouraging and demonstrate that it is possible to develop dynamic one-switch games playable by children with severe motor disabilities. From a group of 8 participants, all of them expressed that they had fun 
playing the GNomon-based mini games, and 7 of them were able to play One Switch Demo and One Switch Ladybugs autonomously. Three of the participant children played also One Switch Invaders and expressed that was the game they liked the most, and two of them wanted to continue playing it after the test.

Currently, a second round of tests is already being carried out with the same group of children to also determine the memorability of GNomon-based mini games. Additionally, future works will consist in improving the framework and in the development of engaging games based on GNomon that can be played by children with disabilities and by ablebody children at the same time. Future developments will also consist in a better tuning of the rotation speed of the clock hands and in extending the NOMON algorithm to support better a dynamically variable number of selectable elements. Lastly, it should also be considered to run long-term studies with larger groups of children with motor disabilities, without cognitive impairments.

\section{ACKNOWLEDGMENT}

The authors would like to thank all the experts of the Regional Health Agency (ASL TO1) that were involved during the development of GNomon and its evaluation. In particular we thank Sara Scotto, Silvana Crivellin and Elisabetta Cane for their time, effort and shared knowledge. Likewise, the authors thank all the children that participated in the study as well as their families.

\section{REFERENCES}

[1] L. Vygotsky, "Play and its role in the mental development of the child," Soviet Psychology, vol. 5, 1967.

[2] I. Granic, A. Lobel, and R. C. M. E. Engels, "The benefits of playing video games." American Psychologist, vol. 69, no. 1, pp. 66-78, 2014.

[3] "Videogames in Europe: Consumer study," Interactive software federation of Europe, Tech. Rep., 2012.

[4] "The state of the worlds children 2013: Children with disabilities," United Nations Childrens Fund (UNICEF), Tech. Rep., 2013.

[5] H. A. Hernandez, Z. Ye, T. N. Graham, D. Fehlings, and L. Switzer, "Designing action-based exergames for children with cerebral palsy," in Proceedings of the SIGCHI Conference on Human Factors in Computing Systems, ser. CHI '13. ACM, 2013, pp. 1261-1270.

[6] C. Green and D. Bavelier, "Learning, attentional control, and action video games," Current Biology, vol. 22, no. 6, pp. R197-R206, 2012.

[7] D. Bavelier, C. S. Green, A. Pouget, and P. Schrater, "Brain plasticity through the life span: learning to learn and action video games," Neuroscience, vol. 35, 2012

[8] J. Angelo, "Comparison of three computer scanning modes as an interface method for persons with cerebral palsy," American Journal of Occupational Therapy, vol. 46, no. 3, pp. 217-222, 1992.

[9] D. Beukelman, Augmentative and alternative communication supporting children and adults with complex communication needs. Paul $\mathrm{H}$. Brookes Pub, 2013.

[10] C. E. Steriadis and P. Constantinou, "Designing human-computer interfaces for quadriplegic people," ACM Trans. Comput.-Hum. Interact., vol. 10, no. 2, pp. 87-118, 2003.

[11] K. Z. Gajos, J. O. Wobbrock, and D. S. Weld, "Improving the performance of motor-impaired users with automatically-generated, abilitybased interfaces," in Proceedings of the SIGCHI Conference on Human Factors in Computing Systems, ser. CHI '08, 2008, pp. 1257-1266.

[12] D. J. C. MacKay, C. J. Ball, and M. Donegan, "Efficient communication with one or two buttons," in Maximum Entropy and Bayesian Methods, ser. AIP Conference Proceedings, vol. 735. American Institute of Physics, 2004, pp. 207-218.
[13] T. Broderick and D. J. MacKay, "Fast and flexible selection with a single switch," PloS one, vol. 4, no. 10, p. e7481, 2009.

[14] M. Belatar and F. Poirier, "Text entry for mobile devices and users with severe motor impairments: Handiglyph, a primitive shapes based onscreen keyboard," in Proceedings of the 10th International ACM SIGACCESS Conference on Computers and Accessibility, ser. Assets '08, 2008, pp. 209-216.

[15] S. Harada, J. O. Wobbrock, J. Malkin, J. A. Bilmes, and J. A. Landay, "Longitudinal study of people learning to use continuous voice-based cursor control," in Proceedings of the SIGCHI Conference on Human Factors in Computing Systems, ser. CHI '09, 2009, pp. 347-356.

[16] R. Ron-Angevin, F. Velasco-lvarez, and S. Sancha-Ros, "Braincomputer interfaces: Proposal of a paradigm to increase output commands," in Intelligent Technologies for Interactive Entertainment, ser. Lecture Notes of the Institute for Computer Sciences, Social Informatics and Telecommunications Engineering, 2012, vol. 78, pp. 18-27.

[17] S. Aced Lopez, F. Corno, and L. De Russis, "Gnomon: Enabling dynamic one-switch games for children with severe motor disabilities," in Proceedings of the 33rd Annual ACM Conference Extended Abstracts on Human Factors in Computing Systems, ser. CHI EA '15. New York, NY, USA: ACM, 2015, pp. 995-1000. [Online]. Available: http://doi.acm.org/10.1145/2702613.2732802

[18] J. L. González Sánchez, N. Padilla Zea, and F. L. Gutiérrez, "From usability to playability: Introduction to player-centred video game development process," in Proceedings of the 1st International Conference on Human Centered Design: Held As Part of HCI International 2009, 2009, pp. 65-74. 